\title{
Evaluation of Prompt Neutron Spectra from Fission of Americium Isotopes
}

\author{
Takaaki Ohsawa* \\ Department of Nuclear Engineering, Faculty of Science and Technology, Kinki University, 3-4-1 \\ Kowakae, Higashi-osaka 577-8502, Japan
}

Received: November 13, 2001; In Final Form: March 12, 2002

Prompt neutron spectra for Am isotopes $\left({ }^{241} \mathrm{Am},{ }^{242 m} \mathrm{Am},{ }^{243} \mathrm{Am}\right)$ were calculated on the basis of a modified version of the Madland-Nix model combined with multimodal fission model. The predicted spectra were found to be in fair agreement with recent data.

\section{Introduction}

With the development of extended burnup of nuclear fuel and incineration of long-lived actinide nuclides, the need for accurate nuclear data for transplutonium isotopes has been rising. The prompt neutron spectra, among others, are required for shielding calculation of transportation casks and reprocessing facilities as well as reactor core calculation. The authors have proposed a method of calculation of the prompt neutron spectra based on the Madland-Nix model ${ }^{1}$ extended to consider the multimodal nature of the fission process. This method was applied to ${ }^{235} \mathrm{U},{ }^{237} \mathrm{~Np}$, and plutonium isotopes with satisfactory results. $^{2-4}$

The method was applied to americium isotopes $\left({ }^{241} \mathrm{Am}\right.$, ${ }^{242 m} \mathrm{Am},{ }^{243} \mathrm{Am}$ ) to obtain the spectra for incident neutron energies up to $5 \mathrm{MeV}$. Difficulties in applying the method to Am isotopes lay in the fact that detailed multimodal analyses of fission have not been made for these nuclides. We solved these problems by making the best use of systematics.

The calculated spectra were compared with experimental data when possible.

\section{Method}

2.1. Mass and Charge Distributions of Fission Fragments. Wang et al. ${ }^{5}$ analyzed empirical data of primary mass distributions for fissioning systems ranging from Ac to Fm and found that the all the distributions were well represented with a superposition of five Gaussian functions with parameters varying smoothly with the mass and excitation energy of the fissioning system. The success of this analysis indicates possible existence of three main modes in fission for the actinides.

The fragment mass distribution for each mode is expressed as a Gaussian function:

$$
G(A, \bar{A}, \sigma)=(2 \pi)^{-1 / 2} \sigma^{-1} \exp \left[-(A-\bar{A})^{2} / 2 \sigma^{2}\right]
$$

where $A$ is the fragment mass number, $\bar{A}$ the average fragment mass number, $\sigma$ the standard deviation of the mass distribution for the fission mode. The total mass distribution $Y\left(A, A_{f}, E_{f}^{*}\right)$ for a fissioning nucleus with excitation energy $E_{f}^{*}$ is written as a superposition of five Gaussians each corresponding to standard$1(S 1)$, standard-2 $(S 2)$ and superlong $(S L)$ modes:

$$
\begin{aligned}
Y\left(A, A_{f}, E_{f}^{*}\right)= & C_{S 1}\left[G\left(A, A_{S 1}, \sigma\right)+G\left(A, A_{f}-A_{S 1}, \sigma\right)\right] \\
& +C_{S 2}\left[G\left(A, A_{S 2}, \mu_{S 2} \sigma\right)+G\left(A, A_{f}-A_{S 2}, \mu_{S 2} \sigma\right)\right] \\
& +C_{S L} G\left(A, A_{f} / 2, \mu_{S L} \sigma\right)
\end{aligned}
$$

where the parameters involved are given as follows for lowenergy fission of actinides 5 :

$$
\begin{aligned}
& C_{S 1}=2.66\left(169.9-N_{f}\right)+0.19\left(A_{f}-232.2\right) E_{f}^{*}, \\
& C_{S 2}=59.3-0.263 N_{f}-0.017\left(A_{f}-235.7\right) E_{f}^{*},
\end{aligned}
$$

$$
\begin{aligned}
C_{S L}= & 0.01 \exp \left(0.46 E_{f}^{*}\right), \\
A_{S 1}= & 141.0-0.053 E_{f}^{*}, \\
A_{S 2}= & 82.3+0.293 N_{f}+0.1 Z_{f}-0.03 E_{f}^{*}, \\
\sigma= & 5.7-0.24\left(149.9-N_{f}\right)+0.12 E_{f}^{*}, \\
\mu_{S L}= & 1.4, \\
\mu_{S 2}= & 1.884-0.0094 N_{f} \\
& +0.267 \exp \left[-\left(N_{f}-142.5\right)^{2}\right] \\
& +0.114 \exp \left[-\left|N_{f}-146.8\right|\right], \\
C= & 100 /\left(C_{S 1}+C_{S 2}+C_{S L} / 2\right) .
\end{aligned}
$$

The quantities $C_{i}$ and $A_{i}$ can be interpreted as the branching ratios and the average fragment masses for a fission mode $i$.

The charge distribution was assumed to be Gaussian with the most probable charge and standard deviation given by Wahl. ${ }^{6}$ The total energy release $E_{R}$ was calculated by using the TUYY mass formula. $^{\text {? }}$

2.2. Total Kinetic Energy of Fission Fragments. Systematics in the empirical data of total kinetic energy (TKE) of fragments for each mode were studied for nuclei in the actinide region on the basis of data analyzed by Fan et al. ${ }^{8}$ It was found that the TKEs for each mode for actinides vary linearly with the Coulomb parameter $Z^{2} / A^{1 / 3}$ of the fissioning nucleus (Figure 1 ). These relations were used to estimate the TKEs for actinides for which the TKEs for each mode are not known.

\section{Results and Conclusion}

Calculations were made for ${ }^{241} \mathrm{Am},{ }^{242 m} \mathrm{Am},{ }^{243} \mathrm{Am}(n, f)$ for incident neutron energies from thermal to $5 \mathrm{MeV}$. The spectra for each fission mode and the synthesized total spectrum for ${ }^{242 m} \mathrm{Am}$ are shown and compared with recent measurement by

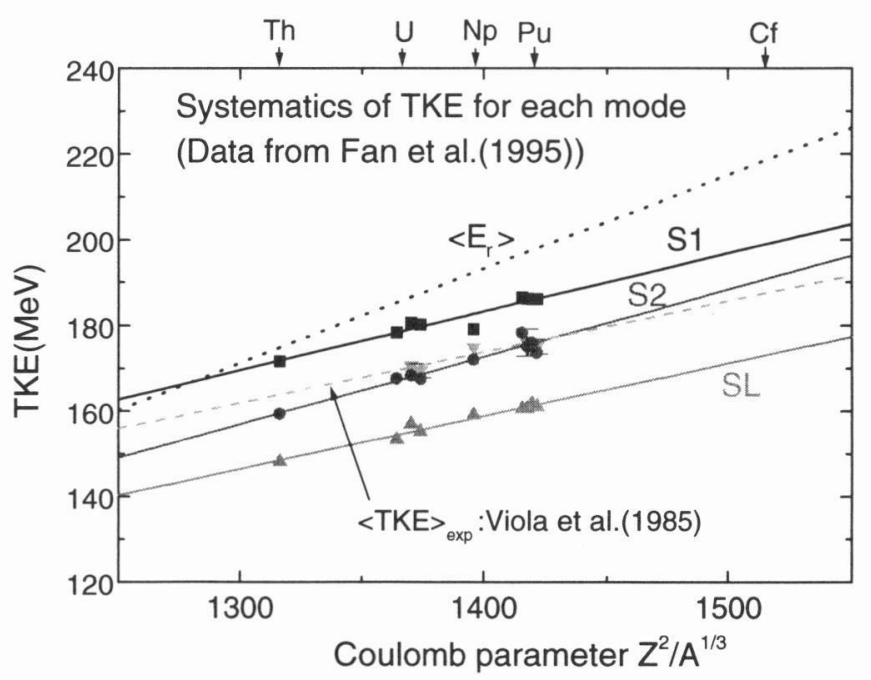

Figure 1. The TKEs for each mode for actinides as a function of the Coulomb parameter $Z^{2} / A^{1 / 3}$ of the fissioning nucleus. 


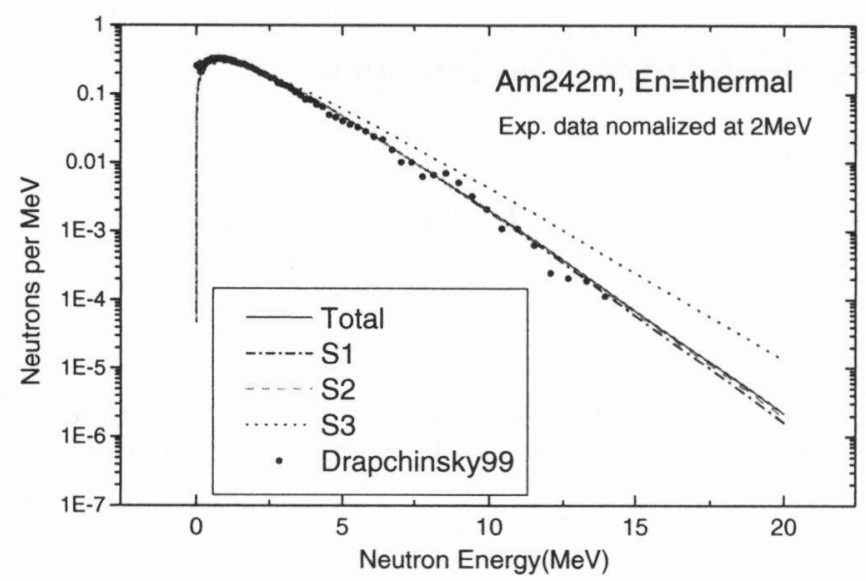

Figure 2. The spectra for each fission mode and the synthesized total spectrum for ${ }^{242 m} \mathrm{Am}$ together with recent measurement by Drapchinsky. ${ }^{9}$

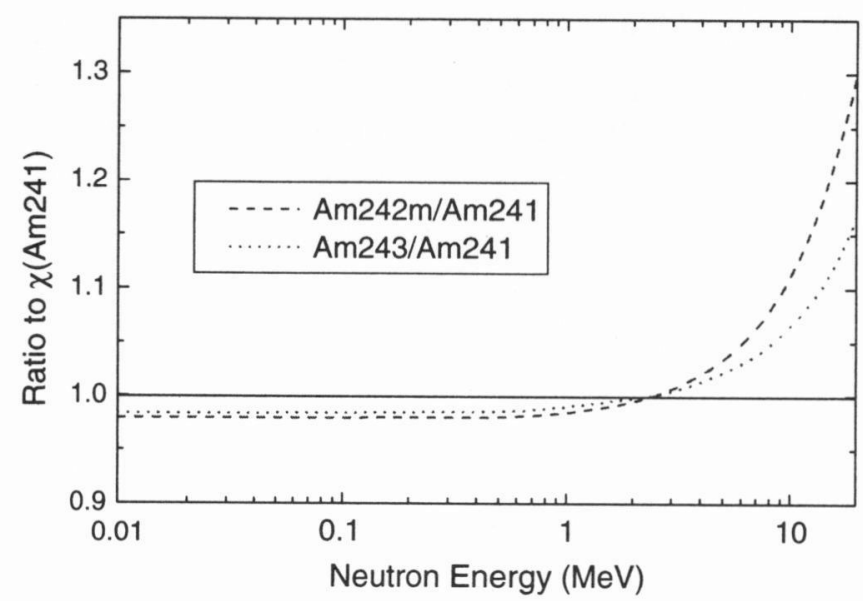

Figure 3. The spectra for ${ }^{242 m} \mathrm{Am}$ and ${ }^{243} \mathrm{Am}$ relative to that for ${ }^{241} \mathrm{Am}$ for the incident energy $E_{n}=3 \mathrm{MeV}$.
Drapchinsky ${ }^{9}$ in Figure 2. It can be seen that the predicted total spectrum agrees fairly well with the measurement, although there are some discrepancies in the region less than $1 \mathrm{MeV}$. This discrepancy may be due to neutron emission before full acceleration of fission fragments, or neutron emission at the instant of scission. This problem is under investigation at present.

The spectra for the three isotopes relative to that for ${ }^{241} \mathrm{Am}$ for the incident energy $E_{n}=3 \mathrm{MeV}$ are compared in Figure 3. It can be seen that, although the differences are not considerable, the spectrum for ${ }^{242 m} \mathrm{Am}$ is the hardest among them and that for ${ }^{243} \mathrm{Am}$ is slightly harder than that for ${ }^{241} \mathrm{Am}$. This tendency can be interpreted from energetics: the neutron binding energy $B_{n}=6.77 \mathrm{MeV}$ for the compound nucleus ${ }^{243} \mathrm{Am}$ is higher than those for other isotopes (5.53 MeV for ${ }^{242} \mathrm{Am}$, and 5.36 MeV ${ }^{244} \mathrm{Am}$ ), and the average total energy release in fission increases with mass number of the fissioning nucleus (207.51 MeV for ${ }^{242} \mathrm{Am}, 208.40 \mathrm{MeV}^{243} \mathrm{Am}$, and $208.42 \mathrm{MeV}$ for $\left.{ }^{244} \mathrm{Am}\right)$. Hence the total excitation energy defined as TXE $=E_{R}+B_{n}+E_{n}-T K E$ increases in the order of ${ }^{241} \mathrm{Am}<{ }^{243} \mathrm{Am}<{ }^{242 m} \mathrm{Am}$.

\section{References}

(1) D. G. Madland and J. R. Nix, Nucl. Sci. Eng. 81, 213 (1982).

(2) T. Ohsawa, T. Horiguchi, and H. Hayashi, Nucl. Phys. A 653, 17 (1999).

(3) T. Ohsawa, T. Horiguchi, and M. Mitsuhashi, Nucl. Phys. A 665, 3 (2000).

(4) T. Ohsawa, Proceedings of the 2000 Symposium on Nuclear Data, Tokai, Nov. 2000, JAERI-Conf 2001-006, 157 (2001).

(5) F. C. Wang and J. M. Hu, J. Phys. G 15, 829 (1989).

(6) A. C. Wahl, At. Data Nucl. Data Tables 39, 1 (1988).

(7) T. Tachibana, M. Uno, M. Yamada, and S. Yamada, At. Data Nucl. Data Tables 39, 251 (1988).

(8) T.-S. Fan, J.-M. Hu, and S.-L. Bao, Nucl. Phys. A 591, 161 (1995).

(9) L. V. Drapchinsky, ISTC 183B-96 (Nov. 1999). 